\title{
Universal health coverage for mental health: the contribution of clinical pharmacists
}

\author{
Filipa Alves da Costa $^{1}$ (D) Derek Stewart ${ }^{2}$ (D)
}

Published online: 13 September 2021

( ) The Author(s), under exclusive licence to Springer Nature Switzerland AG 2021

The patterns of morbidity and mortality have dramatically changed over the last 100 years. Before the 1950s, the most common causes of death were infectious diseases and as public health and medical interventions developed, the shift to noncommunicable diseases (NCDs) became clear. By 2019 , seven out of the ten leading causes of death globally were NCDs, accounting for $74 \%$ of all deaths [1]. Although cardiovascular disease remains the main killer, followed by cancer, the observed and expected further increase in respiratory conditions and in diabetes is worthy of note. Mental health has been gaining attention, mostly because of its impact on disability, with depression having a major role. Globally, mental health conditions account for 1 in 5 years lived with disability, leading to economic losses of more than US\$ 1 trillion per year. By 2019, some mental health conditions, notably Alzheimer's disease and other forms of dementia, were considered the 7th cause of death. Suicide is another major concern, particularly among teenagers and young adults [2]. It has been shown that people living with mental health die prematurely as an indirect result of preventable physical conditions. Mental health conditions often result in loss of capacity to lead productive lives and to integrate in society, leading to situations of vulnerability, including homelessness and substance use, frequently leading to crime. These conditions are especially common for people affected by humanitarian crises and other forms of adversity, including sexual violence.

Even though most mental conditions can be effectively treated, there is a gap in the care offered and in access to care. Aiming to raise awareness about the importance of mental health and the need to ensure access to quality and affordable care for mental health conditions, the World

Filipa Alves da Costa

alvesdacosta.f@gmail.com

1 Faculty of Pharmacy, University of Lisbon, Lisboa, Portugal

2 College of Pharmacy, QU Health, Qatar University, Doha, Qatar
Health Organization launched the Special Initiative for Mental Health (2019-2023): Universal Health Coverage for Mental Health, with the vision that all people will achieve the highest standard of mental health and well-being [3]. This initiative aims to contribute to Sustainable Development Goals (SDGs), particularly SDG target 3.4 .2 by reducing suicide mortality, and target 3.5 by increasing treatment coverage for substance use disorders. To achieve its aims, two strategic pillars have been set: advancing mental health policies, advocacy and human rights; and scaling up interventions and services across community-based, general health and specialist settings.

Aware of this priority, the European Society of Clinical Pharmacy (ESCP), has selected the theme for its annual symposium as, "Clinical pharmacy: working collaboratively in mental health care" [4]. For three days, global experts in clinical pharmacy will gather to discuss updates in pharmacotherapy, notably in dementia, depression, anxiety and psychosis, thus contributing to pillar 2. ESCP is a society committed to promote, support, implement and advance education, practice and research in clinical pharmacy to optimize outcomes for patients and society [5]. Therefore, focus on the most up-to-date evidence gained through robust methodologies is a constant feature of its symposia. Examples of such focus are visible in the 2021 programme, namely by discussing benefit-risk measures applied to mental health and by evaluating trials comparing the benefits of pharmacotherapy and psychotherapy. Involvement of people living with mental health will be a cornerstone of the programme, and this year's Steve Hudson plenary lecture will focus on "Dealing with mental health in excluded minorities", both of which clearly contribute to pillar 1 . The International Journal of Clinical Pharmacy, the affiliated journal to ESCP, encourages its readers to join this event and contribute with their expertise to the discussions.

Funding None. 
Conflicts of interest FAC is an Associate Editor and DS Editor-inChief of the International Journal of Clinical Pharmacy.

\section{References}

1. World Health Organization. The Top 10 causes of Death. Factsheet: December 2020. https://www.who.int/news-room/factsheets/detail/the-top-10-causes-of-death. Accessed 17 Aug 2021

2. World Health Organization. Mental Health. Health Topics. https:// www.who.int/health-topics/mental-health\#tab=tab_1. Accessed 17 Aug 2021]

3. World Health Organization. (2019). The WHO special initiative for mental health (2019-2023): universal health coverage for mental health. World Health Organization. https://apps.who.int/ iris/handle/10665/310981. Accessed 17 Aug 2021. License: CC BY-NC-SA 3.0 IGO

4. ESCP Symposium 2021. https://escpweb.org/virtual-symposium2021. Accessed 17 Aug 2021.

5. European Society of Clinical Pharmacy. Homepage. https://escpw eb.org/. Accessed 17 Aug .2021.

Publisher's Note Springer Nature remains neutral with regard to jurisdictional claims in published maps and institutional affiliations. 